\title{
Performance Analysis of Downlink MIMO WCDMA Systems Using Antenna Selection in Transmitter and MRC Plus LDD in Receiver over Correlated Nakagami-Fading Channels
}

\author{
Siavash Ghavami ${ }^{1}$, Mahmood Mohassel Feghhi' ${ }^{2}$ Bahman Abolhassani ${ }^{2}$ \\ ${ }^{1}$ School of Electrical \& Computer Engineering, University of Tehran, Tehran, Iran \\ ${ }^{2}$ School of Electrical Engineering, Iran University of Science and technology, Tehran, Iran \\ E-mail:s.ghavami@ece.ut.ac.ir,mmohaselfeghhi@ee.iust.ac.ir,abolhassani@iust.ac.ir \\ Received April 9, 2010; revised May 4, 2010; accepted May 12, 2010
}

\begin{abstract}
In this paper bit error rate (BER) performance is analyzed for multiple input-multiple output (MIMO) communications systems using antenna selection in the transmitter, maximal ratio combining (MRC) and linear de-correlating detector (LDD) in the receiver in wide band code division multiple access (WCDMA) downlink channels with correlated Nakagami fading. The MRC maximizes signal to noise ratio of the received signal, then the LDD cancels out multiple access interference (MAI). Theoretical results are validated using computer simulations. Moreover, a pilot based estimation method is proposed to jointly estimate the channel gains and the rows of the LDD operator. Simulation results show that using this proposed method, diversity order is maintained in the receiver. Furthermore, our analysis shows the spectral efficiency degradation due to the pilot based strategy is negligible.
\end{abstract}

Keywords: MIMO, Transmitter Antenna Selection, WCDMA, Maximum Ratio Combining

\section{Introduction}

Wide band code division multiple accesses (WCDMA) has been proposed to satisfy ever-increasing demands for higher data rates, as well as to allow more users to simultaneously access the network [1]. So, it is employed in the third generation mobile networks to provide multimedia services with required qualities. Multiuser detectors (MUDs) are used to detect the desired signal and to simultaneously cancel out interferences coming from cousers in WCDMA systems [2]. In downlink scenario, blind multiuser detectors are proposed for multiple access interference (MAI) cancellation [3], but use of these detectors increases computational complexity of mobile stations (MSs). Another approach for MAI cancellation in downlink multiuser scenario is the precoding method at the base station (BS) [4], but it requires error free links between each MS and the BS, which is not the case in practical scenarios.

Multiple input-multiple-output (MIMO) systems significantly increase system capacity and improve performance $[5,6]$ at the cost of increasing hardware complex- ity by increasing the number of transmitting and receiving antennas. Transmitter antenna selection (TAS) can reduce the cost of multiple antennas and at the same time can retain many advantages of MIMO systems. A combined transmitter antenna selection and maximal ratio combining (MRC) has been proposed in [7]. This method selects the transmitter antenna that maximizes the total received signal power at the receiver. Inactivating other transmitter antennas reduces the hardware complexity; furthermore, this method reduces the number of radio channels used in a MIMO system. Bit error rate (BER) performance of this method has been analyzed in independent and correlated Rayleigh fading channels, respectively in [8] and [9]. As well, recently a BER performance analysis of TAS/MRC has been studied in correlated Nakagami fading channels [10], however it is for a single user scenario in a non CDMA system.

In this paper, the downlink scenario of MIMO WCDMA systems using TAS/MRC plus LDD has been studied. MIMO is a strong tool for capacity increasing and performance improvement. But the limitation in the size of a MS necessitates employing receiver antennas with a 
small distance among them; therefore the correlation among channel gains should be considered even in rich scattering environments. For hardware complexity reduction and keeping diversity order in the base station, TAS in the transmitter is a good candidate, which is considered in this paper. Furthermore for reducing effect of MAI, a linear de-correlating detector (LDD), which is a sub optimum multi user detector, is used in the receiver. The LDD has simple structure with good performance for MAI mitigation [2]. In this paper, the BER performance of a downlink WCDMA system in the correlated Nakagami fading channels is analyzed, and theoretical results are validated using computer simulations. MAI cancellation using MUDs needs to know the user's and co-users' spreading sequences, which increases the complexities of MSs. In this paper, we consider the case in which the links between each MS and the BS are error prone. So, precoding techniques can not be applied. Furthermore, we give up the blind MUDs for their high computational complexities [11]. In this paper, we propose a low complexity pilot based channel estimation method for the joint estimation of the channel gains and the rows of the LDD operator in order to cancel out the MAI. The proposed method does not require the spreading sequences of all active users (which are not available in the MS), as well as the calculation of the inverse cross correlation matrix. So, using this estimation method, the MSs can cancel out the MAI with-out prior knowledge about spreading sequences of co-users by decorrelating users' signals.

The remaining of this paper is organized as follows. In Section 2, system models are considered. BER performance analysis has been presented in Section 3. Joint estimation of channel and the LDD operator is proposed in Section 4. Simulation results are presented in Section 5 in order to validate our performance analysis and evaluate the performance of our proposed joint estimation method. Finally conclusions are presented in Section 6.

\section{System Model}

We consider the downlink scenario of a WCDMA system. The $j$ th antenna of the MS receives signals of $K$ users which have been sent by the $i$ th transmitter antenna in the base station. It is given by

$$
r_{i, j}(t)=\sum_{k=1}^{K} \sum_{l=-\infty}^{\infty} A_{k} d_{k}[l] h_{i, j}\left(t-l T_{s}-\tau_{i, j}\right)+n(t),
$$

where $A_{k}, d_{k}[l], T_{s}$ and $\tau_{i, j}$ are, respectively, the received amplitude, $l$ th data symbol of $k$ th user, symbol period and path delay between $i$ th transmitter antenna and $j$ th receiver antenna and $n(t)$ is additive white Gaussian noise. The $h_{i, j}$ is expressed by:

$$
h_{i, j}(t)=\frac{1}{\sqrt{N}} \sum_{m=0}^{N-1} c_{k}[m] p_{i, j}\left(t-m T_{c}\right),
$$

where $N$ is the processing gain, $T_{c}=T / N$ is the chip time, $p_{i, j}(t)$ is the convolution of three components: the chip pulse shaping waveform, the channel filter between the $i$ th transmitter antenna and the $j$ th receiver antenna (which represents the channel echoes) and the receiver filter, with unit energy. $c_{k}[m]$ is the value of the $m$ th chip of $k$ th user's spreading sequence with $\left|c_{k}[m]\right|=1$. Data symbols $\left\{d_{k}[l]\right\}$ of different users are independent with identical distributions (i.i.d). The channel between the $i$ th transmitter and the $j$ th receiver antenna denotes as $\beta_{i, j}(t)=\alpha_{i, j}(t) e^{j \varphi_{i, j}(t)}$, is assumed to be a quasi-static fading and its envelope $\alpha_{i, j}(t)$ follows Nakagami-m distribution

$$
p_{\alpha_{i, j}}(x)=\frac{2}{\Gamma(m)}\left(\frac{m}{\bar{\delta}}\right)^{m} x^{2 m-1} \exp \left(\frac{-m x^{2}}{\bar{\delta}}\right)
$$

where $\quad m=\bar{\delta}^{2} / E\left[\left(\alpha_{i, j}^{2}-\bar{\delta}\right)^{2}\right] \quad, \quad \bar{\delta}=E\left(\alpha_{i, j}^{2}\right)$, $\Gamma(n)=\int_{0}^{\infty} u^{n-1} e^{-u} d u$. Therefore $\delta_{i, j}=\alpha_{i, j}^{2}$ has gamma distribution,

$$
p_{\delta_{i, j}}(x)=\left(\frac{m}{\bar{\delta}}\right)^{m} \frac{x^{m-1}}{\Gamma(m)} \exp \left(\frac{-m x}{\bar{\delta}}\right) .
$$

\subsection{Transmitter Antenna Selection}

The TAS is performed so that the total received signal power is maximized. Mathematically, it is equivalent to selection antenna such that:

$$
i_{s}=\arg \max _{1 \leq i \leq L_{t}}\left(\sum_{j=1}^{L_{r}}\left|\beta_{i, j}\right|^{2}\right) .
$$

The MS sends $i_{s}$ using only $\left\lceil\log _{2}\left(L_{t}\right)\right\rceil$ bits, where $\lceil\cdot\rceil$ denotes ceiling operation. In performance analysis, channel estimation is assumed to be performed perfectly at the receiver, also the feedback link between the receiver and the transmitter is considered to be perfect and without delay (using forward error correction (FEC) it is possible to send a few flag bits, i.e., $\left\lceil\log _{2}\left(L_{t}\right)\right\rceil$, without error even in error prone link). The size of a Ms limits the use of antenna diversity and this makes the channels correlated. The covariance matrix among channel power gains, e.g., $\boldsymbol{\delta}_{i_{s}}=\left[\begin{array}{lll}\delta_{i_{s}, 1} & \ldots & \delta_{i_{s}, L_{r}}\end{array}\right]^{T}$ in the receiver is 
given by

$$
\boldsymbol{\Omega}=E\left\{\boldsymbol{\delta}_{i_{s}} \boldsymbol{\delta}_{i_{s}}{ }^{H}\right\},
$$

where, $E\{\cdot\}$ and ${ }^{H}$, respectively denote expectation and Hermitian operations.

\subsection{BER Performance Analysis}

In this section, BER performance of TAS/MRC plus LDD in the downlink scenario of WCDMA systems is analyzed. In the following, a bold capital letter denotes a matrix (A), a bold small letter denotes a vector (a), and an unbolded letter denotes a scalar (a or A). Samples of the channel are considered to be constant for each symbol; such that for the $l$ th symbol defined as $\beta_{i, j}[l]=\beta_{i, j}(l T) \quad$. Defining $\mathbf{A}=\operatorname{diag}\left(A_{1}, \ldots, A_{k}\right)$, $\mathbf{C}=\left[\mathbf{c}_{1}^{T}, \ldots, \mathbf{c}_{K}^{T}\right], \quad \mathbf{c}_{k}=N^{-0.5}\left[c_{k}[0], \ldots, c_{k}[N-1]\right]$, $\mathbf{d}[l]=\left[d_{1}[l], \ldots, d_{K}[l]\right]^{T}$ and $\mathbf{n}[l]=\left[n_{j}^{0}[l], \ldots, n_{j}^{N-1}[l]\right]^{T}$, which contains the noise samples in the $j$ th receiver antenna, the received signal form the $i$ th transmitter antenna to the $j$ th receiver antenna is given by

$$
\mathbf{r}_{i, j}[l]=\beta_{i, j}[l] \mathbf{C A d}[l]+\mathbf{n}[l] .
$$

For any linear detector combined with MRC, the decision variable, $\hat{\mathbf{d}}[l]$ is obtained by a linear combination of $\mathbf{r}_{i, j}[l]$, i.e.,

$$
\left.\hat{\mathbf{d}}[l]=\operatorname{sign}\left\{\operatorname{Re}\left(\mathbf{D} \sum_{j=1}^{L_{r}} \beta_{i, j}^{*}[l] \mathbf{r}_{i, j}[l]\right)\right)\right\},
$$

where the matrix D represents the operation of the multiuser detector. For the LDD, D is the Moore-Penrose generalized inverse of the code matrix $\mathbf{C}$ [2], given by

$$
\mathbf{D}_{\mathrm{LDD}}=\left(\mathbf{C}^{\mathrm{T}} \mathbf{C}\right)^{-1} \mathbf{C}^{\mathrm{T}}=\mathbf{R}^{-1} \mathbf{C}^{\mathrm{T}},
$$

where $\mathbf{R}=\mathbf{C}^{\mathrm{T}} \mathbf{C}$ is a $K \times K$ matrix containing the autocorrelation coefficients of the users' spreading codes. In (9), it is assumed that $K$ users' codes are linearly independent, to guarantee the existence of $\mathbf{R}^{-1}$. The receiver output is obtained as follows

$$
\begin{aligned}
\mathrm{v}[l] & =\sum_{j=1}^{L_{r}} \mathbf{R}^{-1} \mathrm{C}^{T} \beta_{i, j}^{*}[l] \mathbf{r}[l] \\
& =\sum_{j=1}^{L_{r}} \mathbf{R}^{-1}\left|\beta_{i, j}[l]\right|^{2} \mathrm{C}^{T} \mathrm{CAd}[l]+\mathbf{R}^{-1} \mathrm{C}^{T} \mathbf{n}[l] \\
& =\sum_{j=1}^{L_{r}}\left|\beta_{i, j}[l]\right|^{2} \mathrm{Ad}[l]+\mathbf{R}^{-1} \mathrm{C}^{T} \mathbf{n}[l] .
\end{aligned}
$$

The $\varepsilon_{k}=\left(\mathbf{R}^{-1}\right)_{k, k} \geq 1$ is the noise enhancement factor produced by the de-correlating operation, therefore noise variance in the receiver side is increased to $\sigma_{n, L D D}^{2}=\varepsilon_{k} \sigma_{n}^{2}$, where $\sigma_{n}^{2}$ is variance of $\mathbf{n}[l]$, which is equal to $N_{0} / 2$.

In the first step of the BER performance analysis, the probability density function (PDF) of maximum channel gains, which has been selected by criterion given by (5) must be obtained, $\delta_{\max }=\max _{1 \leq i \leq L_{t}}\left\{\delta_{i}\right\}$, where $\delta_{i}=\sum_{j=1}^{L_{r}} \delta_{i, j}$. The PDF of $\delta_{\max }$ is obtained as [12]

$$
p_{\delta_{\max }}(x)=L_{t}\left(F_{\delta_{i}}(x)\right)^{L_{t}-1} f_{\delta_{i}}(x)
$$

where $F_{\delta_{i}}(x)$ and $f_{\delta_{i}}(x)$ are respectively, the cumulative distribution function (CDF) and PDF of $\delta_{i} . F_{\delta_{i}}(x)$ and $f_{\delta_{i}}(x)$ for multi user scenario over correlated $\mathrm{Na}$ kagami fading channels are extracted similar to those of a single user scenario over correlated Nakagami fading channels, which proposed in [10].

The TAS in the transmitter and MRC in the receiver increase the received SNR of $k$ th user to

$$
\xi_{k}=\eta_{k} \sum_{i=1}^{L_{r}}\left|\beta_{i_{s} j}\right|^{2}
$$

in which $\eta_{k}=E_{b} /\left(\varepsilon_{k} \sigma_{n}^{2}\right)$, where $E_{b}$ is average energy per bit in the transmitter. For the BPSK modulation the instantaneous BER of the $k$ th user is obtained as $Q\left(\sqrt{2 \xi_{k}}\right)$, where $Q(\cdot)$ is the Q-function. The BER is obtained by integration of instantaneous BER from zero to infinity, as [13]

$$
P_{e, k}=\int_{0}^{\infty} Q\left(\sqrt{2 \xi_{k}}\right) p_{\xi_{k}}\left(\xi_{k}\right) d \xi_{k} .
$$

Since $\xi_{k}$ in (12) is $\eta_{k} \delta_{\max }$, the PDF of $\xi_{k}$ is also obtained similar to $(11)$, e.g., $p_{\xi_{k}}(\cdot)=p_{\delta_{\max }}(\cdot)$. Hence, calculation of integral in (13) can be performed using $p_{\delta_{\max }}(\cdot)$. Calculation of above integral, which is related to BER performance of multi-user scenario, is similar to the calculation of BER performance in a single user scenario, which has been performed in [10]. The final BER is obtained as (14), where, ! denotes the factorial operation and $\left\{\lambda_{l}, 1 \leq l \leq L_{r}\right\}$ are the eigenvalues of the matrix $\sqrt{\Omega} / m$. As well, $\psi_{l r}$ is defined as (15), and the expressions of $a_{j}, b_{j}, c_{j}$ can be obtained using the multinomial theorem [14].

\section{Joint Estimation of Channel Gains and LDD Operator}

In this section, effect of joint estimation of the channel gains and the rows of the LDD operator is investigated. 


$$
\begin{aligned}
& P_{e, k}=L_{t} \sum_{l=1}^{L_{r}} \sum_{r=1}^{m} \frac{\psi_{l r}}{\left(\lambda_{l}\right)^{r} \Gamma(r)} \sum_{j=0}^{\left(L_{t}-1\right)\left(L_{r} \frac{m(m+1)}{2}+1\right)} a_{j}\left(\frac{\lambda_{l}}{b_{j}+\lambda_{l}}\right)^{c_{j}+r} \times\left(c_{j}+r-1 / 2\right) ! \times\left(1-\sqrt{\frac{E_{b} \lambda_{l}}{E_{b} \lambda+\left(b_{j}+\lambda_{l}\right) \varepsilon_{k} N_{0}}}\right)^{c_{j}+r} \\
& \times \sum_{s=0}^{c_{j}+r-1} 2^{-s}\left(c_{j}+r-1+s\right)\left(1+\sqrt{\frac{E_{b} \lambda_{l}}{E_{b} \lambda+\left(b_{j}+\lambda_{l}\right) \varepsilon_{k} N_{0}}}\right)^{s} \\
& \psi_{l r}= \begin{cases}\left(\prod_{k=1, k \neq l}^{L_{r}}\left(1-\frac{\lambda_{k}}{\lambda_{i}}\right)^{-m}\right), & r<m \\
\left.\frac{1}{(m-r) !\left(-\lambda_{l}\right)^{m-r}} \frac{d^{m-r}}{d s^{m-r}}\left(\prod_{k \neq l}\left(1-s \lambda_{k}\right)^{-m}\right)\right|_{s=1 / \lambda_{l}}, & r=m \\
0, & r>m\end{cases}
\end{aligned}
$$

In the downlink scenario of a WCDMA system, each MS knows only its own spreading sequence and doesn't have any knowledge about spreading sequence of any other user, however using the LDD in the receiver needs the knowledge of spreading sequences of all other active users. Moreover, even if a mobile user knows the code matrix of all users (e.g., C) it needs to calculate the inverse cross correlation matrix among spreading sequences of all active users (e.g., $\mathbf{R}^{-1}$ ), which has high computational complexity. Furthermore, the mobile user is allowed to detect only its own data. On the other hand, the total $\mathbf{D}_{\mathrm{LDD}}$ is not required in the mobile. And the $k$ th user requires only the $k$ th row of the $\mathbf{D}_{\text {LDD }}$ denoted by $\mathbf{D}_{L D D}^{k}$ in the $l$ th detected data as can be seen in the following equation

$$
\mathrm{v}_{k}[l]=\sum_{j=1}^{L_{r}} \mathbf{D}_{L D D}^{k}\left|\boldsymbol{\beta}_{i, j}[l]\right|^{2} \mathbf{C A d}[l]+\mathbf{n}^{\prime}[l],
$$

where $\mathbf{n}^{\prime}[l]=\mathbf{D}_{L D D}^{k} \mathbf{n}[l]$. To obtain $\mathbf{D}_{L D D}^{k}$ in the receiver, in this paper, a new method is proposed, in which the base station sends $\mathbf{D}_{L D D}^{k}$ through a pilot signal. In the proposed method, the base station transmits the $\mathbf{D}_{L D D}^{k}$ periodically, whose period is much larger than the symbol time since the number of mobile users changes much slower than the symbol time. Moreover, the base station spreads the pilot signal for transmitting $\mathbf{D}_{L D D}^{k}$ using the $k$ th spreading sequence. This strategy avoids generating interference and maintains the system security since only the $k$ th mobile user can obtain the $\mathbf{D}_{L D D}^{k}$ for detecting its data. The received pilot symbol is as follows:

$$
\mathbf{r}_{j}^{\text {pilot }}=A_{k} \beta_{i_{s}, j}[l] \mathbf{c}_{k} \otimes \mathbf{F}_{\mathbf{k}}+\mathbf{n}^{\prime \prime},
$$

where $\quad \mathbf{F}_{\mathrm{k}}=\mathbf{c}_{k} \otimes \mathbf{D}_{L D D}^{k}, \quad \mathbf{n}^{\prime \prime}=\mathbf{c}_{\mathbf{k}} \otimes \mathbf{n}$ and $\otimes$ denotes element by element multiplications of two vectors. If $A_{k}=1$ then $\mathbf{r}_{j}^{\text {pilot }}$ will be equal to $\beta_{i_{s}, j}[l] \mathbf{F}_{\mathbf{k}}+\mathbf{n}^{\prime \prime}$. Figure 1 shows block diagram of the proposed strategy for joint channel and LDD operator estimation, in which $\mathrm{TS}_{k}$ $[l]$ denotes $l$ th symbol of the training sequence of $k$ th user. Training sequences for LDD operator estimation have been sent in non-overlapping time slots, hence MAI is not produced due to the transmission of pilot signals.

If the pilot symbols length of each user increases, the effect of $\mathbf{n}^{\prime \prime}$ in (17) reduces and a better estimation of $\mathbf{D}_{L D D}^{k}$ can be exploited. Error reduction of $\mathbf{D}_{L D D}^{k}$ estimation is obtained at the expense of increasing the training duration from $N T_{s}$ to $q N T_{s}$, where $q$ is the number of pilot symbols repetition for the desired user.

In the feedback link, $\beta_{i_{s}, j}[l] \mathbf{F}_{k}$ is sent from the mobile user to the base-station to be used for the TAS. Since, $\mathbf{F}_{k}$ is known at the base-station, $\beta_{i_{s}, j}[l]$ can be easily calculated at the base-station. Exactly like the previous section, feedback link is assumed to be error free and without any delay.

Using pilot based strategy for joint estimation of channel gains and LDD operator reduce the spectral efficiency. Hence, effect of using pilot based strategy on the spectral efficiency is analyzed in this section. The spectral efficiency of joint estimation of channel and LDD operator is defined as,

$$
\eta=\frac{T I B}{T T B}=1-\frac{q K \lambda}{R_{b}},
$$




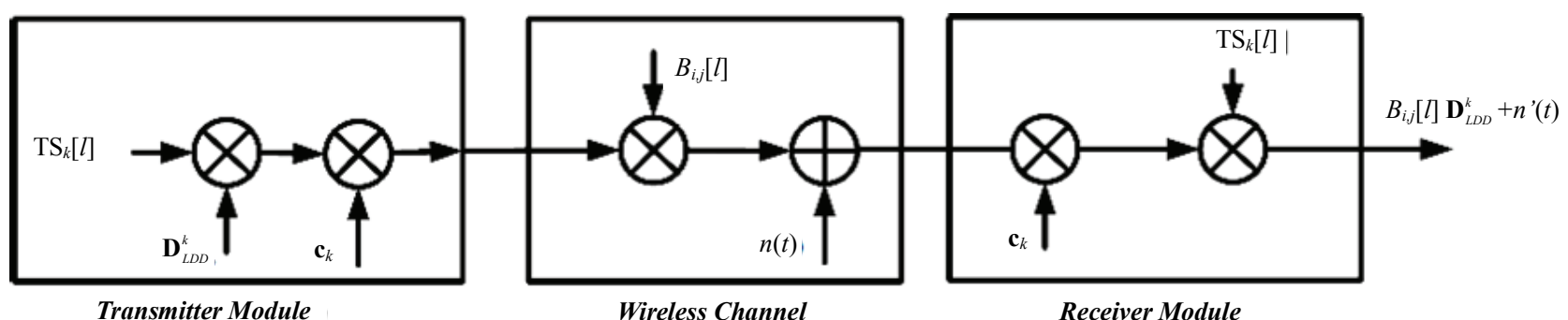

Figure 1. Block diagram of proposed joint channel estimation and LDD operator method.

where, TIB and TTB are transmitted information bits and total transmitted bits, respectively; also, $\lambda$ and $R_{b}$ are variation rate of number of arriving user to the cell and bit rate, respectively. The spectral efficiency of the proposed method is analyzed for practical values of $q$,

\section{$K$ and $\lambda$ in the next Section.}

In the next section, the currently explained algorithm is used for the joint estimation of channel and the LDD operator. Also, the bit error probability performance of this method is evaluated and compared with the bit error probability of the same system with the perfect estimation of channel and the LDD operator.

\section{Simulation Results}

In this section, we validate our analysis, equation (14), using computer simulations. We simulate the TAS/MRC plus LDD scheme in flat Nakagami correlated fading channels. Furthermore, the effect of the proposed method for joint estimation of channel and the LDD operator is studied on the BER performance of TAS/MRC plus LDD system.

The base station transmits data with equal amplitudes for each of $K=25$ users. Random sequence with length of 63 has been used for short spreading codes. A rectangular pulse shaping waveform $p(t)$ is used for shaping filter in the transmitter side. The type of modulation is BPSK. Simulations are performed for a MIMO system with 2 transmitter antennas and 3 receiver antennas over correlated Nakagami flat fading channels. The normalized correlation antenna matrix is the same as the one given in [9], it is given by a $3 \times 3$ matrix denoted by $\Omega=\left[\Omega_{1}, \Omega_{2}, \Omega_{3}\right]$, where $\Omega_{1}=\left[\begin{array}{lll}1 & 0.727 & 0.913\end{array}\right]^{T}$, $\Omega_{2}=\left[\begin{array}{lll}0.727 & 1 & 0.913\end{array}\right]^{T}$ and $\Omega_{3}=\left[\begin{array}{lll}0.913 & 0.913 & 1\end{array}\right]^{T}$.

Figure 2 shows the BER of the TAS/MRC plus LDD in the aforementioned Nakagami correlated fading channels for $m=1,2$ and 3 . It is obvious that our analytical result given by (14) is validated by computer simulations. Moreover, the system with TAS/MRC plus LDD has a better performance so that it has approximately $3.5 \mathrm{~dB}$ SNR gain relative to that of the MRC plus LDD without the TAS at $P_{e, k}=10^{-4}$ with the diversity order $m=1$.

Figure 3 shows the effect of proposed joint estimation of channel gains and the LDD operator on the performance of the TAS/MRC plus LDD with a training sequence having length of either 25 or 50 , which needs once $(q=1)$ or twice $(q=2)$ repetition of $\mathbf{D}_{\mathrm{LDD}}$ elements, respectively. From Figure 3, it is obvious that the diversity order remains almost constant since the slope of the three

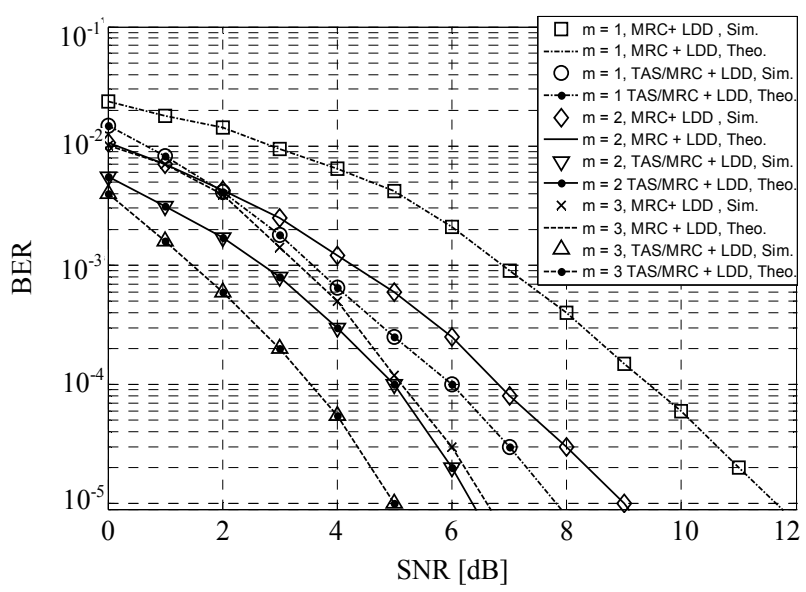

Figure 2. BER performance of MRC plus LDD and TAS/ MRC plus LDD.

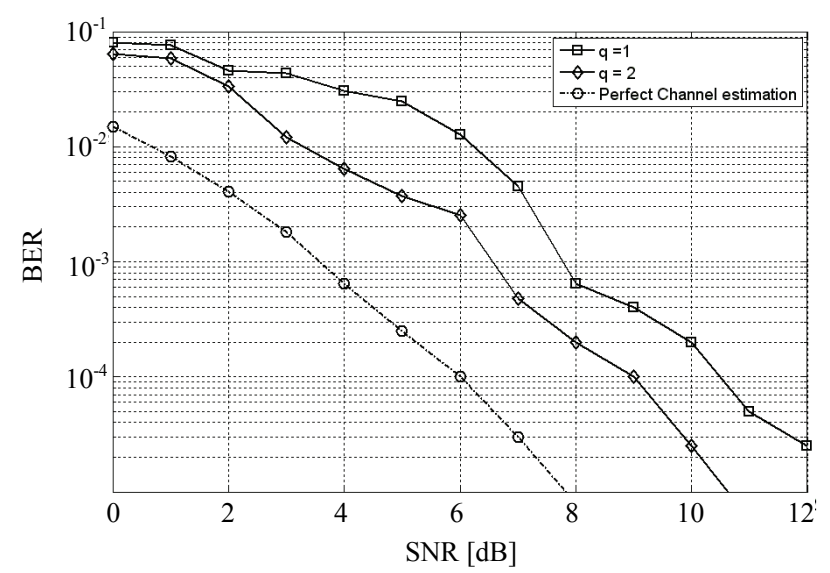

Figure 3. BER performance of TAS/MRC plus LDD for $\mathbf{m}$ $=1$ with imperfect channel estimation. 
curves are approximately the same, and there is only 4.5 $\mathrm{dB}$ or $3 \mathrm{~dB}$ loss in the SNR at $P_{e, k}=10^{-4}$ due to using the proposed joint estimation of channel and the LDD operator with training sequence whose length is 25 or 50 , respectively. It is notable that by increasing $q$ the SNR loss reduces.

The mean square error (MSE) of the proposed joint estimation of channel and the LDD operator is defined as,

$$
M S E=E\left[\left\|\left(\mathbf{D}_{L D D}\right)_{k,:}-\left(\hat{\mathbf{D}}_{L D D}\right)_{k,:}\right\|^{2}\right]
$$

where $\left(\hat{\mathbf{D}}_{L D D}\right)_{k,:}$ is $k$ th row of the estimated LDD opera- tor, $E\{\cdot\}$ denotes expectation operator and $\left\|\left(\mathbf{D}_{L D D}\right)_{k,:}-\left(\hat{\mathbf{D}}_{L D D}\right)_{k,:}\right\|^{2}$ denotes square norm of matrix. Figure 4 shows $M S E$ of the proposed joint estimation of channel and the LDD operator over AWGN, in terms of number of pilot repetition $q$, for different values of SNR. It is obvious that by increasing $q, M S E$ of $\left(\mathbf{D}_{L D D}\right)_{k \text {, }}$ estimation is reduced.

Figure 5(a) shows the $\eta$ in terms of the $q$ for different values of active users in the cell with traffic variation of $\lambda=10 \mathrm{user} / \mathrm{sec}$ and $R_{b}=5 \mathrm{Mbps}$. It is obvious even with $q=50$ bits and $k=90$ users, the efficiency is greater than 0.99. Figure 5(b) is similar to Figure 5(a) with $\lambda=20 \mathrm{user} / \mathrm{sec}$. It can be seen with $q=50$ bits and $k=90$ users, the efficiency is greater than 0.98. Figure 5(c) and 5(d) are similar to Figure 5(a) with $\lambda=50 \mathrm{user} / \mathrm{sec}$ and $\lambda=100 \mathrm{user} / \mathrm{sec}$, respectively.

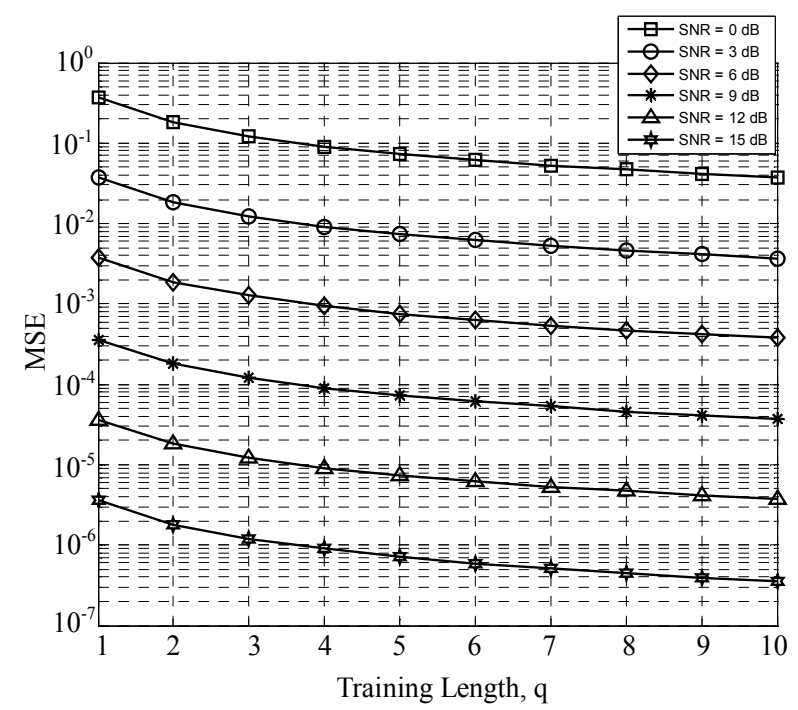

Figure 4. Mean Square error of joint estimation of channel and the LDD operator in terms of training length.

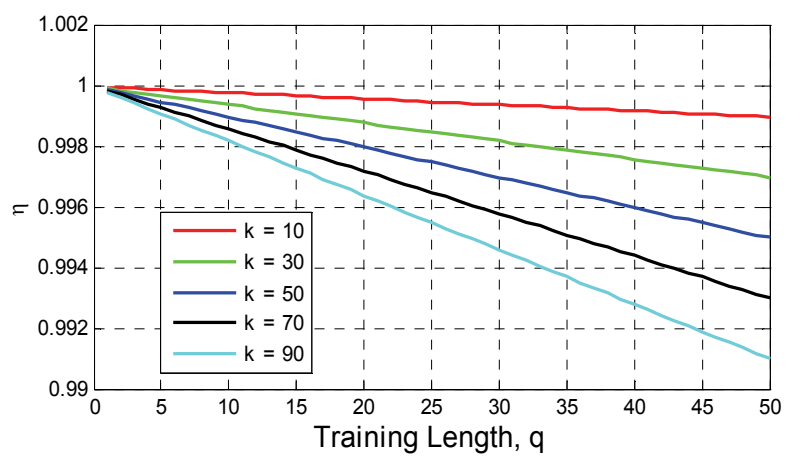

(a) $\lambda_{1}=10 \mathrm{user} / \mathrm{sec}$

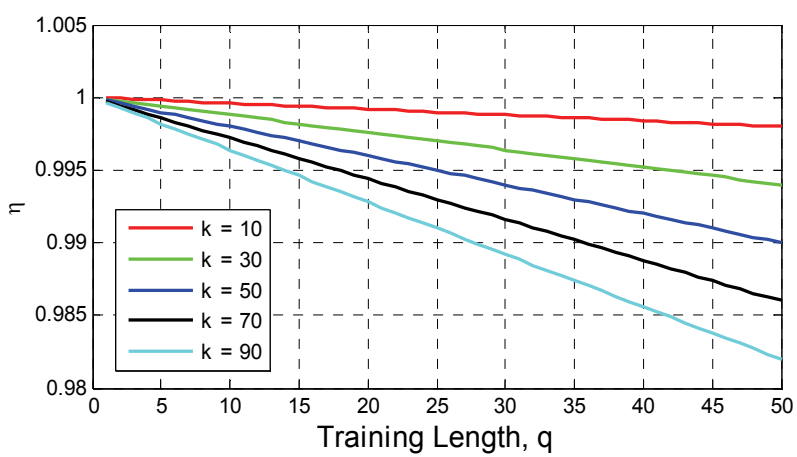

(b) $\lambda_{2}=20$ user/sec

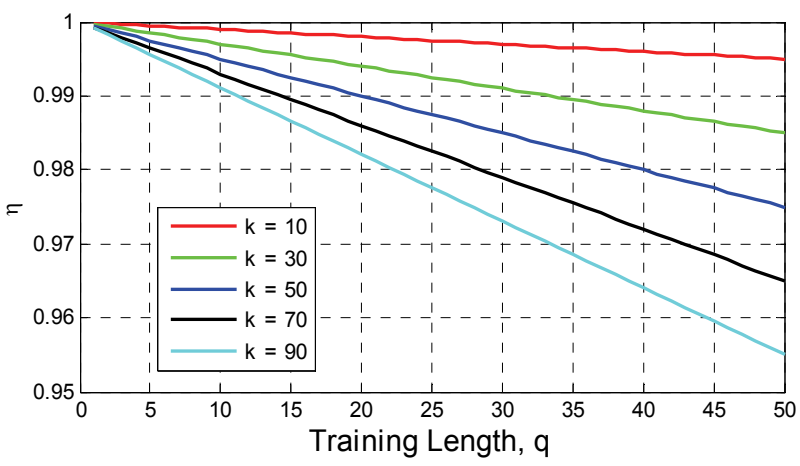

(c) $\lambda_{3}=50$ user/sec

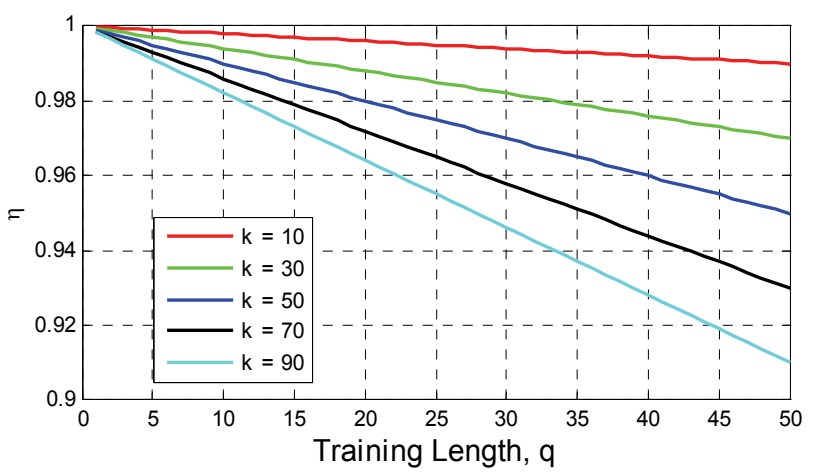

(d) $\lambda_{4}=100 \mathrm{user} / \mathrm{sec}$

Figure 5. Efficiency in terms of number of pilot symbols repetition for different number of active users in cell with (a) variation of input traffic of $\lambda_{1}=10 \mathrm{user} / \mathrm{sec}$, (b) $\lambda_{2}=20$ user $/ \mathrm{sec}$, (c) $\lambda_{3}=50$ user/sec and (d) $\lambda_{4}=100$ user/sec. 


\section{Conclusions}

In this paper, the BER performance analysis of the TAS/ MRC plus LDD is performed for the downlink WCDMA network in correlated Nakagami fading channels. Equation (14), derived in the analysis, was validated using computer simulations. Also a pilot based channel estimation method is proposed for joint estimating of the channel gains and the LDD operator. Simulation results show that with joint estimation of channel gains and the LDD operator, diversity order is kept in the receiver. Moreover, our analysis shows that the spectral efficiency degradation due to using the pilot based strategy for joint estimation of channel gains and LDD operator is negligible.

\section{References}

[1] L. B. Milstein, "Wideband Code Division Multiple Access," IEEE Journal on Selected Areas in Communications, Vol. 18, No. 8, August 2000, pp. 1344-1354.

[2] S. Verdu, "Multiuser Detection," Cambridge University Press, Cambirdge, 1998.

[3] X. Wang and H. V. Poor, "Blind Multiuser Detection: A Subspace Approach," IEEE Transactions on Information Theory, Vol. 44, No. 2, March 1998, pp. 677-690.

[4] B. R. Vojcic and W. M. Jang, "Transmitter Precoding in Synchronous Multiuser Communications," IEEE Transactions on Communications, Vol. 46, No. 10, October 1998, pp. 1346-1355.

[5] E. Telatar, "Capacity of Multi-Antenna Gaussian Channels," European Transactions on Telecommunications, Vol.
10, No. 6, 1999, pp. 585-595.

[6] G. J. Foschini and M. J. Gans, "On Limits of Wireless Communications in a Fading Environment When Using Multiple Antennas," Wireless Personal Communications, Vol. 6, No. 3, March 1998, pp. 311-335.

[7] S. Thoen, L. Van der Perre, B. Gyselinckx and M. Engels, "Performance Analysis of Combined Transmit-SC/ Receiver-MRC," IEEE Transactions on Communications, Vol. 49, No. 1, 2001, pp. 5-8.

[8] Z. Chen, J. Yuan and B. Vucetic, "Analysis of Transmit antenna Selection/Maximal-ratio Combining in Rayleigh Fading Channels," IEEE Transactions on Vehicular Technology, Vol. 54, No. 4, 2005, pp. 1312-1321.

[9] L. Yang, D. Tang and J. Qin, "Performance of Spatially Correlated MIMO Channel with Antenna Selection," IEE Ectronics Letters, Vol. 40, No. 20, 2004, pp. 1281-1282.

[10] B. Y. Wang and W. X. Zheng, "Exact BER of Transmitter Antenna Selection/Receiver MRC over Spatially Correlated Nakagami-Fading Channels," IEEE Conference on Circuits and Systems, Kos, May 2006, pp. 1370-1373.

[11] Y. Yao and H. V. Poor, "Blind Detection of Synchronous CDMA in Non-Gaussian Channels," IEEE Transactions on Signal Processing, Vol. 52, No. 1, January 2004, pp. 271-279.

[12] A. Papoulis, "Probability, Random Variables and Stochastic Processes," 4th Edition, McGraw-Hill, New York, 2001.

[13] J. G. Proakis, "Digital Communications," 4th Edition, McGraw-Hill, New York, 2001.

[14] R. L. Graham, D. B. Knuth and O. Patashnik, "Concrete Mathematics,” Addison-Wesley, New York, 1989. 